\title{
Diabetic cardiomyopathy - heart disease in diabetes
}

\author{
Alena ADAMIKOVA ${ }^{1}$, Jiri BAKALA ${ }^{2}$, Jana PATEROVA ${ }^{2}$, Martin SLABAK ${ }^{3}$, \\ Stepan SVACINA ${ }^{4}$
}

${ }^{1}$ Diabetes Center, Bata Regional Hospital, Zlín, Czech Republic

${ }^{2}$ Department of Nuclear Medicine, Bata Regional Hospital, Zlín, Czech Republic

${ }^{3}$ Cardiovascular Center, Bata Regional Hospital, Zlín, Czech Republic

${ }^{4}$ First Faculty of Medicine, Charles University, Prague, Czech Republic

\section{Email address:}

adamikova@bnzlin.cz(A. ADAMIKOVA)

\section{To cite this article:}

Alena ADAMIKOVA, Jiri BAKALA, Jana PATEROVA, Martin SLABAK, Stepan SVACINA. Diabetic Cardiomyopathy - Heart Disease in Diabetes. Clinical Medicine Research. Vol. 2, No. 4, 2013, pp. 89-93. doi: 10.11648/j.cmr.20130204.20

\begin{abstract}
Diabetic cardiomyopathy is defined as a finding of systolic and diastolic left ventricular dysfunction, myocardial dilation and left ventricular hypertrophy without the presence of macroangiopathy and hypertension. Causes include metabolic changes, myocardial fibrosis, microangiopathy as well as cardiovascular autonomic neuropathy leading to sympathetic denervation and alteration of myocardial perfusion. It comprises abnormalities in the control of heart rate as well as central and peripheral vascular dynamics. The diagnosis of diabetic cardiomyopathy affects significantly the prognosis in patients with diabetes. Echocardiography and nuclear medicine methods are used for diagnosis.
\end{abstract}

Keywords: Cardiovascular Autonomic Neuropathy, Diabetic Cardiomyopathy, Myocardial Scintigraphy, Echocardiography

\section{Introduction}

Diabetic cardiomyopathy is a multifactorial disease. Its etiology involves metabolic changes in the myocytes, myocardial fibrosis as well as microangiopathy, and is manifested by left ventricular dysfunction without the presence of coronary artery disease and hypertension. Diabetic neuropathy is one of the most common late complications of diabetes. After ten years of diabetes its prevalence is up to $100 \%$. Neuropathy affects both proximal and distal sensory and motor nerves as well as the autonomic nervous system. Diabetic autonomic neuropathy has a clear impact on survival and quality of life in patients with diabetes. It contributes to the development of diabetic cardiomyopathy. In a study by Ziegler et al. [1]-year mortality was five times higher for patients with cardiovascular autonomic dysfunction than in patients without this disease. A dominant role in the etiology of neuropathy is played by hyperglycemia, which affects the development of oxidative stress, impaired vascular blood supply and metabolism in nerves. According to Vinik et al. [2] cardiovascular autonomic neuropathy leads to physical exercise intolerance, orthostatic hypotension, and silent ischemia and even to painless myocardial infarction.

\section{Diabetic Cardiovascular Complications}

\subsection{Cardiovascular Autonomic Diabetic Neuropathy}

As a form of neuropathy cardiovascular autonomic neuropathy (CAN) covers abnormalities in the control of heart rate and of the central and peripheral vascular dynamics. Cardiac denervation syndrome is manifested by reduced heart rate variability, at-rest and fixed tachycardia, prolongation of the QT interval shown on ECG, as well as impaired diurnal variation in blood pressure with an insufficient decrease at night. Orthostatic hypotension is another symptom - with a fall in systolic blood pressure when standing up of more than $30 \mathrm{mmHg}$ and diastolic pressure of more than $10 \mathrm{mmHg}$. If the fall is not accompanied by changes in heart rate response, it may be associated with reduced secretion of catecholamines in a very severe form CAN. Cardiac workload intolerance is caused by a decreased response of heart rate and blood pressure to exercise, a reduction in cardiac output during exercise, a reduced ejection fraction, systolic dysfunction and diastolic filling restrictions. Diabetic patients with $\mathrm{CAN}$ are also at a greater risk during surgery using general 
anesthesia. CAN can be manifested by an impaired response to hypercapnia and hypoxia, a tendency to intraoperative hypothermia, a need for higher doses of vasopressor agents, as well as a greater risk of a postoperative hypertonic crisis. Patients with diabetes have also been reported to have more frequent gastrointestinal motility disorders and a tendency to gastroparesis during the postoperative period. Also, the occurrence of silent myocardial ischemia may be associated with the presence of CAN. A metaanalysis by Vinik et al. [2] studies with silent ischemia in diabetic patients established a $28 \%$ (p $<0.001)$ presence of CAN, while patients with CAN also had statistically significant increased mortality $(p<0.0001)$. Ewing tests were use in the diagnosis of CAN for many years to identify the response of heart and blood pressure to deep breathing, a Valsava maneuver and orthostasis. Currently, spectral analysis of heart rate is used to diagnose CAN. Nuclear medicine techniques may be applied to see adrenergic innervation of the myocardium using ${ }^{123} \mathrm{I}$ MIBG (metaiodobenzylguanidine). The treatment of cardiovascular autonomic neuropathy, in addition to improving glycemic control, is mainly symptomatic.

\subsection{Diabetic Cardiomyopathy}

Diabetic cardiomyopathy (DCM) is defined as ventricular dysfunction in patients with diabetes, which is caused by coronary atherosclerosis and hypertension. It shows specific functional and structural changes in the myocardium leading to left ventricular dysfunction, and even hypertrophy. Left ventricular hypertrophy is associated with sudden death [3]. Pathophysiology DCM is complex. Hyperglycemia, excess free fatty acids and insulin resistance have an impact on cardiac myocytes. Hyperglycemia is associated with increased formation of advanced glycation end products (AGEs). Furthermore, there is increased production of reactive oxygen species, inflammatory cytokinins and expression of adhesion molecules. The increased tendency to thrombosis is associated with the activation of platelets and reduced fibrinolysis [4]. Intramyocardial causes also include microangiopathy. Additional pathophysiological processes include cardiovascular autonomic neuropathy, whereby impaired vascular tone results in inadequate myocardial perfusion, and changes in preload and afterload occur during each heartbeat [5].

\section{Case Report}

A 45-year-old female patient treated for diabetes type 1 since 1985. From the beginning the disease was controlled by an intensified basal/bolus regimen. In 1989 sensomotoric diabetic distal polyneuropathy was diagnosed. In 1998 an additional health problem appeared - diabetic diarrhea. In 2003 the patient underwent scintigraphy, and a gastric emptying study identified autonomous neuropathy in the region of the gastrointestinal tract. Additional complications appeared in 2011 - incipient non- proliferative retinopathy and cataracta corticonuclearis. The patient's laboratory results (Table 1) showed inadequate parameters of long-term control HbA1c 9.1\% (DCCT, 77 $\mathrm{mmol} / \mathrm{mol}$ ), while other findings were normal. Cardiovascular risk factor high-sensitivity CRP $1.5 \mathrm{mg} / 1$ were normal. Exercise testing proved exercise intolerance, resting tachycardia and low blood pressure values of 110/70 $\mathrm{mmHg}$. Due to the presence of other forms of neuropathy we considered a diagnosis of autonomic neuropathy within the patient's cardiovascular system, which belongs to the pathophysiological causes of diabetic cardiomyopathy.

Table 1. Laboratory Date

\begin{tabular}{|c|c|c|}
\hline Methods & Laboratory Data & Normal level \\
\hline Glucose & $7.3 \mathrm{mmol} / 1$ & $3.9-5.5 \mathrm{mmol} / \mathrm{l}$ \\
\hline Total cholesterol & $5.45 \mathrm{mmol} / 1$ & $<5.0 \mathrm{mmol} / 1$ \\
\hline $\mathrm{HbAlc}$ & $9.1 \%=77 \mathrm{mmol} / \mathrm{mol}$ & $20-42 \mathrm{mmol} / \mathrm{mol}$ \\
\hline HDL & $2.0 \mathrm{mmol} / 1$ & $>1.27 \mathrm{mmoml} / 1$ \\
\hline Creatinine & $67 \mu \mathrm{mol} / 1$ & $49-90 \mu \mathrm{mol} / 1$ \\
\hline LDL & $3.25 \mathrm{mmol} / 1$ & $<3.0 \mathrm{mmol} / 1$ \\
\hline Urea & $5.6 \mathrm{mmol} / \mathrm{l}$ & $2.9-8.2 \mathrm{mmol} / \mathrm{l}$ \\
\hline Triglycerides & $0.95 \mathrm{mmol} / 1$ & $<1.7 \mathrm{mmol} / 1$ \\
\hline Uric acid & $183 \mu \mathrm{mol} / 1$ & $140-340 \mu \mathrm{mol} / \mathrm{l}$ \\
\hline High sensitivity CRP & $1.5 \mathrm{mg} / 1$ & $<3.0 \mathrm{mg} / 1$ \\
\hline Cystatin C & $0.76 \mathrm{mg} / 1$ & $<0.96 \mathrm{mg} / 1$ \\
\hline Apo B & $0.86 \mathrm{~g} / 1$ & $<0.99 \mathrm{~g} / 1$ \\
\hline Microalbuminuria & $1.8 \mathrm{mg} / 1$ & $<20 \mathrm{mg} / 1$ \\
\hline GF & $2.12 \mathrm{ml} / \mathrm{s} / 1.73 \mathrm{~m} 2$ & $\begin{array}{l}1.5-2.5 \\
\mathrm{ml} / \mathrm{s} / 1.73 \mathrm{~m} 2\end{array}$ \\
\hline
\end{tabular}

HbA1c glycosylated haemoglobin, HDL high density lipoprotein, LDL low density lipoprotein, Apo B apolipoprotein B, GF glomerular filtration

Resting echocardiography showed no signs of left ventricular failure. LVEF was $73 \%$, diastolic dysfunction was not present, there were no signs of valvular abnormalities and/or pulmonary hypertension, the left atrium was normal. The left ventricle TEI index (total ejection isovolumic index) was 0.16 (standard $\leq 0.4$ ), diabetic cardiomyopathy was not confirmed.

In addition, we performed an assessment of adrenergic cardiac innervation using a ${ }^{123}$ I MIBG (iodine-123-metaodobenzylguanidine) scan by means of planar scintigraphy (30 minutes and 4 hours), as well as a SPECT 40 minutes after application. Heart-to-mediastinal ratio and wash-out rate were calculated. A SPECT scan was performed at 40 minutes. Planar images were used to monitor the wash-out rate in the mediastinum vs. heart tissues. In addition, the authors evaluated segment abnormalities using a SPECT 
study. We found significant hypoperfusion of the anterior segment, with an H/M index of 1.85. The wash-out rate was accelerated everywhere, most of all from the parts in the area of reduced blood flow.

Exercise perfusion testing and resting myocardial perfusion scintigraphy including semiquantitative evaluation were complemented with a gated resting and exercise SPECT, followed by image reconstruction in Short ax., H-long ax., V-long ax. views. At-rest images failed to demonstrate any focal myocardial perfusion impairment in the left ventricle. Following exercise, the images showed a region of anterior hypoperfusion, which was not evident at rest. After exercise there was a decrease in ejection fraction, as well as a deterioration of ESV and EDV (Table 2, Fig. 1). Thus, the findings suggested exercise-induced systolic (as well as possibly diastolic) left ventricular dysfunction, and along with autonomic neuropathy, the presence of diabetic cardiomyopathy. Transient ischemic left ventricular dilatation (TID) shown by a stress myocardial perfusion SPECT scan is a marker of the presence of severe coronary artery disease $[6,7]$. The patient had a normal TID value as well as negative exercise testing result. Therefore, coronary angiography was not indicated.
Table 2. Evaluation of the exercise myocardial SPECT

\begin{tabular}{llll}
\hline \multicolumn{4}{l}{ Evaluation of the exercise myocardial SPECT } \\
\hline STRESS & $70(\mathrm{ED}=71$, & REST & \\
EF (\%) & $\mathrm{ES}=21)$ & EF (\%) & $76(\mathrm{ED}=74$, \\
SSS (\%) & 7 & SRS & 2 \\
SMS & 10 & RMS & 5 \\
STS & 4 & RTS & 0 \\
PER & 2.85 & PER & 3.23 \\
PFR & 2.95 & PFR & 3.73 \\
PRF2 & 1.75 & PRF2 & 1.06 \\
MFR/3 & 1.61 & MFR/3 & 2.05 \\
TTPF & 151 & TTPF & 155 \\
TID $=\mathbf{0 , 9 0}$ & & & \\
BPM & 72 & BPM & 66 \\
\hline
\end{tabular}

EF: ejection fraction (\%), ED: end-diastolic volume (ml), ES: end-systolic volume $(\mathrm{ml})$, SSS: summed stress score $7 \%(0-3 \%$ negative $4-8 \%$ unclear, $9-13 \%$ probability of finding $25 \%,>13 \%$ probability of finding $50 \%$ ), SRS: summed rest score, SMS: stress motion score, STS: stress thickening score, RMS: rest motion score, RTS: rest thickening score, PER: peak ejection rate, PFR2: peak filling rate (second diastolic phase), MFR/3: mean filling rate/3, TTPF: time to peak filling, BPM: beat per minute, TID: transient ischemic dilatation

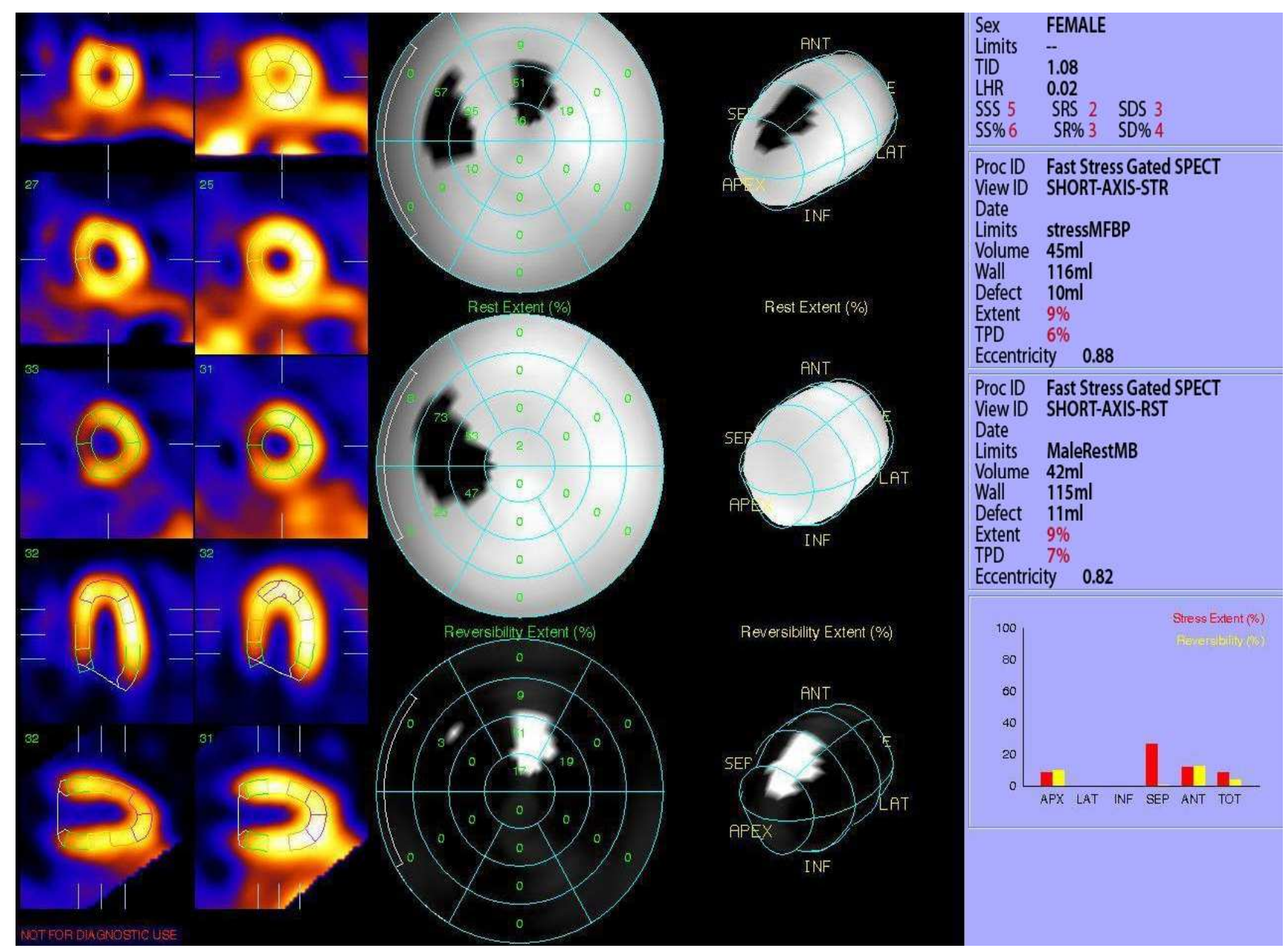

Figure 1. Examination of adrenergy cardiac innervations using ${ }^{123} I$ MIBG (iodine-123-meta-odobenzylguanidine) by the method of planar scintigraphy and calculation of heart/mediastinal index. Archive of Department of Nuclear Medicine, Bata Regional Hospital, Zlin, Czech Republic. 


\section{Discussion}

Diagnostic methods dealing with diabetic cardiomyopathy mainly use non-invasive techniques that show left ventricular hypertrophy and a reduction in both systolic and diastolic myocardial function. Non-invasive diagnostic echocardiography is the principal technique. An index which evaluates the volume of left ventricular ejection is the total isovolumic index (TEI), i.e. the myocardial performance index (MPI). The index is calculated $(a-b / b)$ from the ratio of difference in time intervals from the end to the start of the transmitral flow (a) and the left ventricular ejection time (b) to the ventricular ejection time (b), and the TEI index may also be expressed by the formula (IVCT + IVRT/ET), from the ratio of the sum of the isovolumic contraction time (IVCT) and the isovolumic relaxation time (IVRT) and left ventricular ejection time (ET) [8,9]. A standard TEI (MPI) vale is $\leq$ 0.40 . Diastolic dysfunction is defined as a state, where the ventricle with no increase in pressure in the left atrium is not filled so as to ensure sufficient cardiac output. Diastolic dysfunction is caused by: impaired relaxation, increased ventricular stiffness, short duration of diastole, lack of atrial contribution. Its prevalence increases with age and is more common in women. Diastolic dysfunction has a better prognosis than systolic. In a study in diabetic patients without macrovascular complications the TEI index was significantly and independently associated with the presence of diabetic cardiomyopathy [10]. Higher levels of the TEI index were associated with an increased propensity to ventricular arrhythmias. Several studies deal with assessment of the TEI index during dobutamine stress echocardiography. Nørager et al. [11] demonstrated an increase in the index in a group of 50 patients with newly diagnosed myocardial infarction on echocardiography with a low dose dobutamine compared with 25 healthy volunteers. Changes in the TEI index provided a quantitative measurement of total functional reserve of the left ventricle in patients with myocardial infarction. Ling et al. [12] used the TEI (MPI) index as a supplement to assess and analyze cardiac wall motion in the detection of myocardial ischemia during dobutamine atropine stress echocardiography. Additional echocardiographic parameters monitored in diabetic cardiomyopathy include LAVI, i.e. the ratio of left atrial volume and surface area (left atrial volume index - standard up to $28 \mathrm{ml} / \mathrm{m} 2$ ), the ratio in the mitral flow $\mathrm{E} / \mathrm{A}$, the systolic left ventricular function is assessed using the LV ejection fraction, standard $\geq 55 \%$. The patient had TEI index values within the standard.

Another non-invasive method for differential diagnosis of cardiomyopathy is to perform post-exercise and resting perfusion myocardial scintigraphy using technetiumlabeled a MYOWIEW heart scan using SPECT with a semiquantitative evaluation, and an additional gated stressrest SPECT test, which allows us to define, in addition to a perfusion problem, possible kinetics disorders at rest and after exercise, as well as impaired left ventricular wall thickening at rest and after exercise. A method for diagnosing autonomic neuropathy in the cardiovascular area is receptor scintigraphy, especially one using ${ }^{123} \mathrm{I}$ MIBG, allowing us to see any reduction in cardiac adrenergic innervation [13, 14]. Adrenergic innervation images and their quantification are obtained following the application of ${ }^{123} \mathrm{I}$ labeled metaiodobenzylguanidine (MIBG). Patient images are obtained using planar and SPECT scans. Evaluation consists of calculating ROI (region of interest) density of adrenergic receptors on the heart compared to a mediastinal reference area; radiopharmac wash-out is assessed. The output includes two parameters: heart/mediastinum and accelerated radiopharmac wash-out from the heart. In a study performed by Bakala et al. (15) the H/M (heart / mediastinum) the ratio in patients without diabetes and without CHD was 3.88, in diabetic patients with no coronary heart disease 2.4 , and diabetic patients with an evidence of neuropathy 1.1 ; the wash-out in patients with diabetes was faster by $44 \%$, in patients without diabetes by $20 \%$. Adrenergic innervation imaging using ${ }^{123} \mathrm{I}$ MIBG allows us to detect autonomic neuropathy within the cardiovascular system. [16]. The ADMIRE-HF study [17, $18,19,20]$ conducted in 96 centers in Europe, the USA and Canada showed that Adrenergic innervation imaging using ${ }^{123}$ I MIBG makes it possible to stratify patients at high risk of heart failure, arrhythmia and sudden death. The study demonstrated during a two-year monitoring of mortality that patients with $\mathrm{H} / \mathrm{M}$ levels between 1.6 to 1.2 had a 10 fold higher risk than patients with $\mathrm{H} / \mathrm{M}$ values above 1.6

Our study patient's resting images during myocardial scintigraphy failed to demonstrate any focal myocardial perfusion abnormality within the left ventricle. The images following ergometry exercise revealed anterior hypoperfusion, which was not evident at rest. After exercise there was a decrease in the ejection fraction as well as deterioration in ESV and EDV. The H/M index was 1.85 . The wash-out rate from the region of reduced perfusion was accelerated. Thus, the findings gave evidence of stressinduced systolic (i.e. possibly also diastolic) left ventricular dysfunction, and together with autonomic neuropathy, the presence of diabetic cardiomyopathy.

The therapy of DCM is based on improved diabetes control, e.g. the use of an insulin infusion pump, control of hypertension using ACE inhibitors, angiotensin II receptor blockers, maintenance of atrium contractility, reduction of neurohumoral activation, as well as improvement of myocardial metabolism by means of heart failure therapy.

\section{Conclusion}

The diagnosis of cardiomyopathy has a significant effect on patient prognosis. DCM is associated with an increased risk of heart failure and sudden death. Adrenergic 
innervation imaging and its quantification using ${ }^{123} \mathrm{I}-\mathrm{MIBG}$ scintigraphy facilitate the diagnosis of cardiovascular autonomic neuropathy. Additionally, the use of exercise perfusion scintigraphy can assist us in early detection of disease dynamics, and thus in therapy specification.

\section{References}

[1] D. Ziegler, C. P. Zentai and S. Perz S. Prediction of mortality using measures of cardiac autonomic dysfunction in the diabetic and nondiabetic population The MONICA/KORA Augsburg Cohort Study. Diabetes Care 2008; 31, pp. 556-561.

[2] A. I. Vinik, RE. Maser and BD. Mitchell. Diabetic autonomic neuropathy. Diabetes Care 2003; 26 (5), pp. $1553-1579$

[3] P. Spirito, P. Bellone, K. M. Harris, P. Bernabo, P. Bruzzi and B. J. Maron. Magnitude of left ventricular hypertrophy and risk sudden death in hypertrophic cardiomyopathy. $\mathrm{N}$ Engl J Med 2000; 342, pp. 1778-1785.

[4] C. Voulgari, D. Papadogiannis and N. Tentolouris. Diabetic cardiomyopathy: from the pathophysiology of cardiac myocytes to current diagnosis and management strategies. Vascular Health and Risk Management 2010; 6, pp. 883-903.

[5] S. Lacigova, J. Meinlova and J. Gruberova. The heart of patient with type 1 diabetes. Vnitř Lék 2010; 56(5), pp. 418426.

[6] M. Petretta,W. Acampa and S. Daniele. Transient ischemic dilation in SPECT myocardial perfusion imaging for prediction of severe coronary artery disease in diabetic patients. J Nucl Cardiol 2012; doi: 10. 1007/s12350-0129642-6.

[7] A. Adamikova, J. Bakala, J. Bernatek, J. Rybka and S. Svacina. Transient ischemic dilation ratio (TID) correlates with $\mathrm{HbAlc}$ in patients with diabetes type 2 with proven myocardial ischemia according to exercise myocardial SPECT. Ann Nucl Med 2006; 20(9), pp. 615-621.

[8] C. Tei. New non-invasive index for combined systolic and diastolic ventricular function. J Cardiol 1995; 26, pp. 135136.

[9] C. Tei, L. H. Ling, D. O. Hodge, K. R. Bailey, J. K. Oh and J. K.Tajik. New index of combined systolic and diastolic myocardial performance: a simple and reproducibile measure of cardiac function: a study in normal and dilated cardiomyopathy. J Cardiol 1995; 26, pp. 357-366.
[10] C. Voulgari, N. Tentolouris and D. Papadagiannis. Increased left ventricular arrhytmogenicity in metabolic syndrome and relationship with myocardial performance, risk factors for athrerosclerosis, and low-grade inflammation. Metabolism 2010; 59, pp. 159-165.

[11] B. Nørager, M. Husic, J. E. Møller and K. Egstrup. The myocardial performance index during low-dose dobutamine echocardiography in control subjects and patients with a recent myocardial infarction: a new index of left ventricular functional reserve? Journal of the American Society of echocardiography 2004; 17(7), pp. 732-738.

[12] L. H. Ling, C. Tei, R. B. McCully, K. R. Bailey, J. B. Seward and P. A. Pellikka. Analysis of systolic and diastolic time intervals during dobutamie-atropine stress echocardiography: Diagnostic potencial of the doppler myocardial performance index. Journal of the American Society of Echocardiography 2001; 14(10), pp. 978-986.

[13] S. Murashima, K. Takeda, K. Matsumura, K. Yamakado, H. Sakuma and T. Nakagawa. Increased lung uptake of Iodine123-MIBG in diabetics with sympathetic nervous dysfunction. J Nucl Med 1998; 39(2), pp. 334-338.

[14] A. Scott and P. L. Kench. Cardiac autonomic neuropathy in diabetic patient: does ${ }^{123} \mathrm{I}-\mathrm{MIBG}$ imaging have role to play in early diagnosis? J Nucl Med Technol 2004; 32, pp. 66-71.

[15] J. Bakala, J. Bernatek, P. Kurfürst and A. Adamikova. Comparison cardiac adrenergic innervation between patients with and without diabetes mellitus using 123IMIBG planar scintigraphy and SPECT method. [abstract] Cas Lék Ces 2011; 150(2), pp. 116-117.

[16] S. Nagamachi, S. Jinnouchi, T. Kurose, R. Nishii, K. Kawai and S. Futami. Serial chase in 123I-MIBG myocardial scintigraphy in non-insulin-depender diabetes melllitus. Ann Nucl Med 2002; 16(1), pp. 33-38.

[17] D. Agostini, H. J. Verberne, M. Hamon, AF. Jacobson and A. Manrique. Cardiac 123I-MIBG scintigraphy in heart failure. J Nucl Med Mol Imaging 2008; 52(4), pp. 369-77.

[18] D. Agostini, I. Carrrio and H. J. Verberne. How to use myocardial 123I-MIBG scintigraphy in chronic heart failure. Eur J Nucl Med Mol Imaging 2009; 36, pp. 555-559.

[19] A. F. Jacobson, J. Lombard, G. Banerjee and P. G. Camici. 123I-mIBG scintigraphy to predict risk for adverse cardiac outcomes in heart failure patients: designe of two prospective multicenter international trials. J Nucl Card 2009; 16(1), pp. 113-21.

[20] S. Hughes. ACC: ADMIRE-HF: new imaging test helps better define risk in heart failure. 2009; http://www.medscape.com/viewarticle/590516. 